\title{
Théâtres et amphithéâtres : outils de romanisation en Maurétanie?
}

\section{Adeline Pichot}

\section{(2) OpenEdition}

\section{Journals}

Édition électronique

URL : http://journals.openedition.org/edl/113

DOI : $10.4000 /$ edl. 113

ISSN : 2296-5084

\section{Éditeur}

Université de Lausanne

\section{Édition imprimée}

Date de publication : 15 mai 2011

Pagination : 171-192

ISBN : 978-2-940331-25-3

ISSN : 0014-2026

\section{Référence électronique}

Adeline Pichot, "Théâtres et amphithéâtres : outils de romanisation en Maurétanie ? », Études de lettres [En ligne], 1-2 | 2011, mis en ligne le 15 mai 2014, consulté le 18 décembre 2020. URL : http:// journals.openedition.org/edl/113; DOI : https://doi.org/10.4000/edl.113 


\section{THÉÂTRES ET AMPHITHÉÂTRES: OUTILS DE ROMANISATION EN MAURÉTANIE?}

Les théâtres et les amphithéâtres faisaient partie de la parure monumentale des grandes villes des Maurétanies césarienne et tingitane. Avec leur architecture spécifique et leurs spectacles, ils permettaient d'assimiler la population à la culture romaine et représentaient la puissance impériale. Tous les habitants s'y retrouvaient pour se détendre et participer au culte rendu aux dieux romains et à l'empereur.

\section{Introduction}

La chute de Carthage en 146 av. J.-C. marque le début de la colonisation romaine en Afrique du Nord. Pour parvenir à ses fins, Rome créa de nouveaux centres urbains, qu'elle peupla des vétérans de l'armée, et continua le développement des agglomérations indigènes préexistantes. Comme le décrit Strabon dans son recueil sur la géographie ${ }^{1}$, l'urbanisation est le facteur par excellence de la romanisation ou de l'assimilation des populations dans la sphère culturelle romaine.

Les spectacles, éléments essentiels de la société urbaine, trouvèrent tout naturellement leur place en Afrique. Il existe de très nombreux témoignages architecturaux, épigraphiques et iconographiques de leur importance, dont une partie est présentée ici. Pour les provinces d'Afrique proconsulaire et de Numidie, de nombreuses monographies et

I. Strabon, Géographie 3.2. 
synthèses ont déjà été publiées ${ }^{2}$, mais ce travail doit encore être effectué pour les Maurétanies césarienne et tingitane.

Après une présentation rapide des Maurétanies, nous proposons un panorama des théâtres et des amphithéâtres, suivi d'un exposé des problématiques liées à ces monuments: pour quels types de spectacles étaient-ils conçus? Quels monuments antérieurs leur ont servi d'exemples? Lesquels ont-ils ensuite inspirés? Quelle fut leur importance dans la romanisation des populations indigènes? Nous nous attarderons ensuite sur la compréhension du monument de Lixus, ainsi que sur l'importance de ces édifices comme moyen de diffusion de la culture romaine.

\section{Les Maurétanies romaines}

L'Afrique romaine correspond à quatre provinces antiques (fig. 1): l'Afrique proconsulaire, la Numidie, la Maurétanie césarienne et la Maurétanie tingitane. Elle est délimitée à l'ouest par l'océan Atlantique et à l'est par le golfe de la Grande Syrte. Au-delà se situe la Cyrénaïque, qui par son héritage politique et culturel se rattache à la partie orientale de l'Empire romain, de langue et de civilisation grecques ${ }^{3}$. Si le front de mer s'étend sur $2500 \mathrm{~km}$, la pénétration romaine à l'intérieur des terres est moins importante, elle varie entre 50 et $300 \mathrm{~km}$.

Entourée par plusieurs façades littorales, l'Afrique communiquait principalement par navire avec le reste du monde romain. La Proconsulaire, la Numidie et la Césarienne produisaient les deux tiers du blé nécessaire pour Rome, suite aux confiscations des domaines agricoles africains sous Néron ${ }^{4}$. Leur réseau routier et maritime était subordonné à l'exportation de cette denrée essentielle. Les voies terrestres de Césarienne mènent toutes à des ports importants comme Portus Magnus, Caesarea ou Icosium (Alger).

2. Par exemple J.-C. Lachaux, Théâtres et amphithéâtres d'Afrique proconsulaire.

3. Elle n'a jamais été rattachée à l'Afrique, mais réunie administrativement à la Crète, dès l'époque augustéenne.

4. Pline l'Ancien, Histoire naturelle 18.7. 


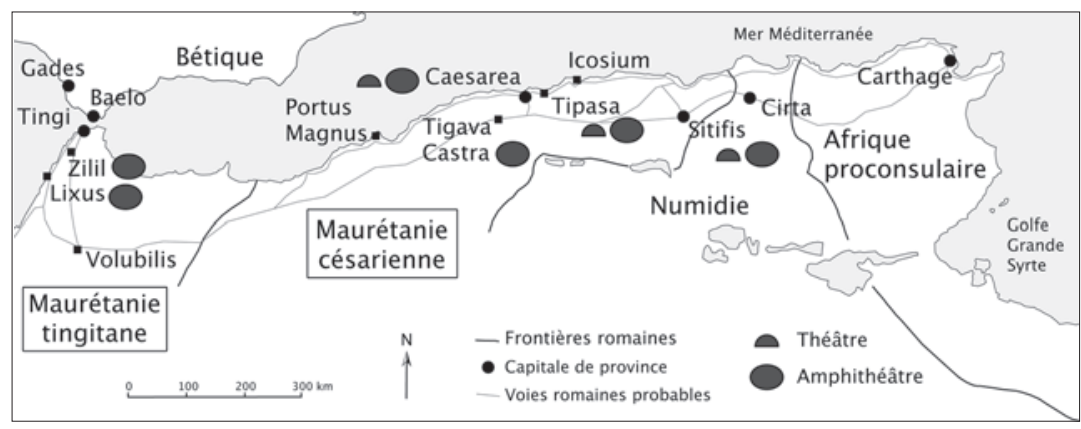

Fig. 1 - Plan de l'Afrique romaine.

Les preuves d'une participation de la Tingitane à l'annone sont plus rares et elle y contribuait probablement en faible quantités. En revanche, elle exportait de l'huile, des salaisons de poisson, des bêtes sauvages et des bois rares comme le thuya. Tingi entretenait un commerce actif avec l'Espagne, les circulations dans le détroit de Gibraltar étaient faciles et nombreuses. La colonie claudienne de Baelo, tête de pont vers l'Afrique, fut créée en face même de Tanger. La route maritime de Baelo à Tingi est exactement dans le prolongement de la voie terrestre Tingi-Volubilis, et la ligne joignant par mer Gades à Lixus poursuit le parcours continental le long de la côte. Les contacts entre la province de Tingitane et celle de Bétique (sud de l'Espagne) étaient très étroits ${ }^{6}$.

Les Maurétanies étaient gouvernées par des procurateurs de rang équestre, placés sous l'autorité de l'empereur. Ils commandaient des contingents d'auxiliaires qui constituaient une force permanente de dissuasion. En cas de nécessité, la légion III Augusta basée en Numidie pouvait être transportée sur les secteurs menacés ${ }^{7}$. La création de la Maurétanie sitifienne, avec Sitifis comme capitale, fut décidée par Dioclétien afin de mieux organiser les prélèvements fiscaux. Datée traditionnellement de 288 de notre ère, elle aurait eu lieu un peu plus tard

5. Des greniers ont été identifiés récemment à Thamusida et la mention de fiscalia frumentaria dans l'édit de Caracalla laisse penser que cette province livrait des céréales. A. Pichot, Les édifices de spectacle des Maurétanies romaines, p. 30.

6. L. Harmand, L'Occident romain, p. 430.

7. F. Decret, M. Fantar, L'Afrique du nord dans l'Antiquité, p. 192. 
en $303^{8}$. Dans notre étude, nous ne la distinguons pas de la Césarienne, puisqu'il s'agit d'un découpage tardif.

Dans les cités africaines, le sénat local s'efforçait d'offrir à la population un mode de vie à la romaine qui permettait d'obtenir de la part de l'administration impériale une promotion pour la ville, par exemple un titre de colonie honoraire qui donnait à tous les citoyens des droits égaux à ceux de Rome et permettait de payer moins d'impôts. Le sénat local finançait directement ou profitait de l'évergétisme ${ }^{9}$ pour faire construire les lieux de vie publique comme le forum, la basilique, les temples, le marché ou les établissements de détente (thermes, bibliothèques et édifices de spectacle). Les témoignages d'évergésies sont rares en Césarienne et totalement absents en Tingitane. Il faut donc se référer au reste de l'Afrique pour mieux comprendre ce phénomène.

Dans la société romaine, le corps et l'âme devaient pouvoir s'adonner à l'otium, le temps libre qui offre la paix, le calme pour l'esprit et la chair, voire une inaction empreinte de paresse et d'oisiveté. Il s'oppose au negotium, l'absence de loisir comme les activités militaires, politiques ou commerciales ${ }^{10}$. Au départ, cette notion de loisir était liée aux fêtes religieuses, les feriae organisées pour honorer les dieux avec des prières, des processions, des sacrifices et des ludi. Ces derniers étaient constitués de courses de chars et de représentations théâtrales. Une fois les Maurétanies intégrées dans l'Empire romain, les municipes et les colonies ont adopté le calendrier qui impliquait la célébration de jeux en l'honneur des dieux (ludi publici). Le statut des colonies romaines et des municipes latins prévoyait en outre la création annuelle de fêtes romaines ${ }^{11}$. Toutes les cités pourvues de monuments de spectacle reçurent le titre de colonie, sauf Tigava Castra situé à proximité du municipe de Tigava. Elles devaient donc organiser de nombreux spectacles chaque année. Sans oublier les jours de fêtes impériales qui étaient décidés par la chancellerie romaine et imposés par sénatus-consulte à toutes les provinces. On ne connaît malheureusement pas le nombre exact de jours

8. J.-P. Laporte, «Une inscription de Saldae et la date de séparation des Maurétanies Césarienne et Sitifienne», p. 1119.

9. Les promesses de dons des candidats aux élections.

IO. S. Barthélemy, D. Gourevitch, Les loisirs des Romains, p. XXXI.

II. Voir le détail de ces jours de fête d'après la loi d'Urso, une colonie de vétérans fondée par Jules César en Bétique, dans Ch. Hugoniot, «Peut-on écrire que les spectacles furent un facteur de romanisation en Afrique du Nord?», p. 243. 
fériés dans ces provinces, mais la vie d'un habitant des Maurétanies était ponctuée par ces réjouissances.

\section{Les monuments}

Actuellement, trois théâtres et cinq amphithéâtres sont connus en Maurétanie (fig. 1). Les deux édifices de Sitifis sont seulement attestés par l'épigraphie ${ }^{12}$. Les données sur l'amphithéâtre de Tigava Castra sont trop lacunaires pour être publiées, ce monument n'ayant jamais été fouillé. Les théâtres et les amphithéâtres de Caesarea et de Tipasa, comme les monuments de Lixus et de Zilil, sont conservés et ont fait l'objet de recherches sur le terrain. Nous avons regroupé leur description par ville, en commençant par ceux de Maurétanie césarienne.

\subsection{Caesarea}

Les premiers habitants du site de Caesarea étaient des Phéniciens, qui fondèrent une modeste colonie appelée Iol. Le témoignage le plus ancien du site est une lampe punique datée du VIe siècle av. J.-C. ${ }^{13}$. Lorsque Juba II arriva au pouvoir, placé sur le trône par Auguste en 25 av. J.-C., il entreprit la construction d'une nouvelle capitale pour son royaume. Il se conformait à la tradition en conservant l'emplacement punique, mais il perpétuait le mythe des rois fondateurs. Il donna à sa ville le nom de Caesarea en l'honneur d'Auguste ${ }^{14}$. Ce choix symbolisait à la fois un attachement politique et religieux, et le lien indéfectible entre le patronus et son client. A la bienveillance et protection du premier répondait la dépendance totale du second, chacun ayant un devoir envers l'autre. Cette coutume s'enracine dans l'Orient grec. Elle est moins présente dans les régions occidentales ${ }^{15}$, mais Caesarea est le parfait exemple de ce type de relation.

I2. Le théâtre: CIL VIII 8438, l'amphithéâtre: CIL VIII 8482 et 8507, $A E$ 1928, p. 38 et $A E 1949$, p. 258.

I3. N. Benseddik, T. W. Potter, La fouille du forum de Cherchel.

I4. Ph. Leveau, "L'urbanisme des princes clients d'Auguste», p. 353.

I5. M. Coltelloni-Trannoy, Le royaume de Maurétanie sous Juba II et Ptolémée (25 av. J.-C. -40 apr. J.-C.), p. 145. 
Juba II a fait construire les édifices publics caractéristiques d'une ville romaine: forum, thermes, théâtre, amphithéâtre et cirque, qui se répartissent le long des axes de circulation. Les éléments architectoniques retrouvés laissent imaginer de nombreux autres monuments, palais et temples, encore enfouis sous la ville actuelle. Sous Claude (41-54), la ville reçut la dignité de colonie romaine à titre honoraire ${ }^{16}$ et devint en importance la deuxième ville d'Afrique du Nord après Carthage.

Le théâtre ${ }^{17}$, construit entre 25 et 15 av. J.-C., est le plus ancien d'Afrique (fig. 2). Juba II a entrepris sa construction dès son arrivée au pouvoir, pour orner rapidement sa capitale d'un théâtre de type italien. Son plan s'inspire certainement du théâtre de Pompée à Rome, avec un temple au sommet de la cavea $^{18}$, mais le chantier du théâtre de Marcellus commencé par César a pu aussi l'influencer. Le théâtre était un outil de propagande impériale efficace, à la fois par son architecture typiquement "romaine» et par les représentations que l'on y donnait ${ }^{19}$.

Le théâtre faisait partie du projet d'urbanisme initial de la ville. Il formait la limite sud du forum et se trouvait au cœur de la cité. Plusieurs statues ont été retrouvées lors des fouilles. Elles ornaient certainement le mur de scène: deux muses colossales, une tête en marbre coiffée d'une dépouille d'éléphant qui est une personnification de l'Afrique (fig. 3) et une statue colossale d'Auguste ${ }^{20}$. Le spectateur pouvait être impressionné par ce riche décor et la représentation impériale.

La scène, l'orchestre et les trois premiers gradins du bas ont disparu lors de la mise en place d'une arène, au $\mathrm{II}^{\mathrm{e}}$ ou au III ${ }^{\mathrm{e}}$ siècle apr. J.-C. Les parodoi servaient de porta triumphalis, par laquelle sortaient les combattants vainqueurs, et de porta libitinensis, qu'empruntaient les morts et les blessés. Ce théâtre-amphithéâtre est un exemple très rare en Afrique. Cette transformation n'est pas due à un appauvrissement de la ville ${ }^{21}$,

I6. Elle ne reçut pas de colons italiens. Cf. Pline l'Ancien, Histoire Naturelle 5.20 et N. Benseddik et al., Cherchel, p. 10.

17. Cavea adossée de $45 \mathrm{~m}$ de diamètre, avec 27 gradins pouvant contenir environ 6300 personnes.

I8. Soubassement de $6 \mathrm{~m}$ sur 4,80 m. Voir l'étude architecturale de J. Vérité dans C. Sintes, Y. Rebahi, Algérie antique, p. 277 sq. et F. Sear, Roman theatres, p. 104.

19. G. Ch. Picard, "La date du théâtre de Cherchel», p. 52.

20. S. Gsell, Cherchel, antique Iol-Caesarea, p. 88 sq.

2I. S. Gsell supposait que la transformation avait suivi les dévastations de Firmus, en 371 ou 372 apr. J.-C. Cette solution se serait avérée plus économique que la restauration 

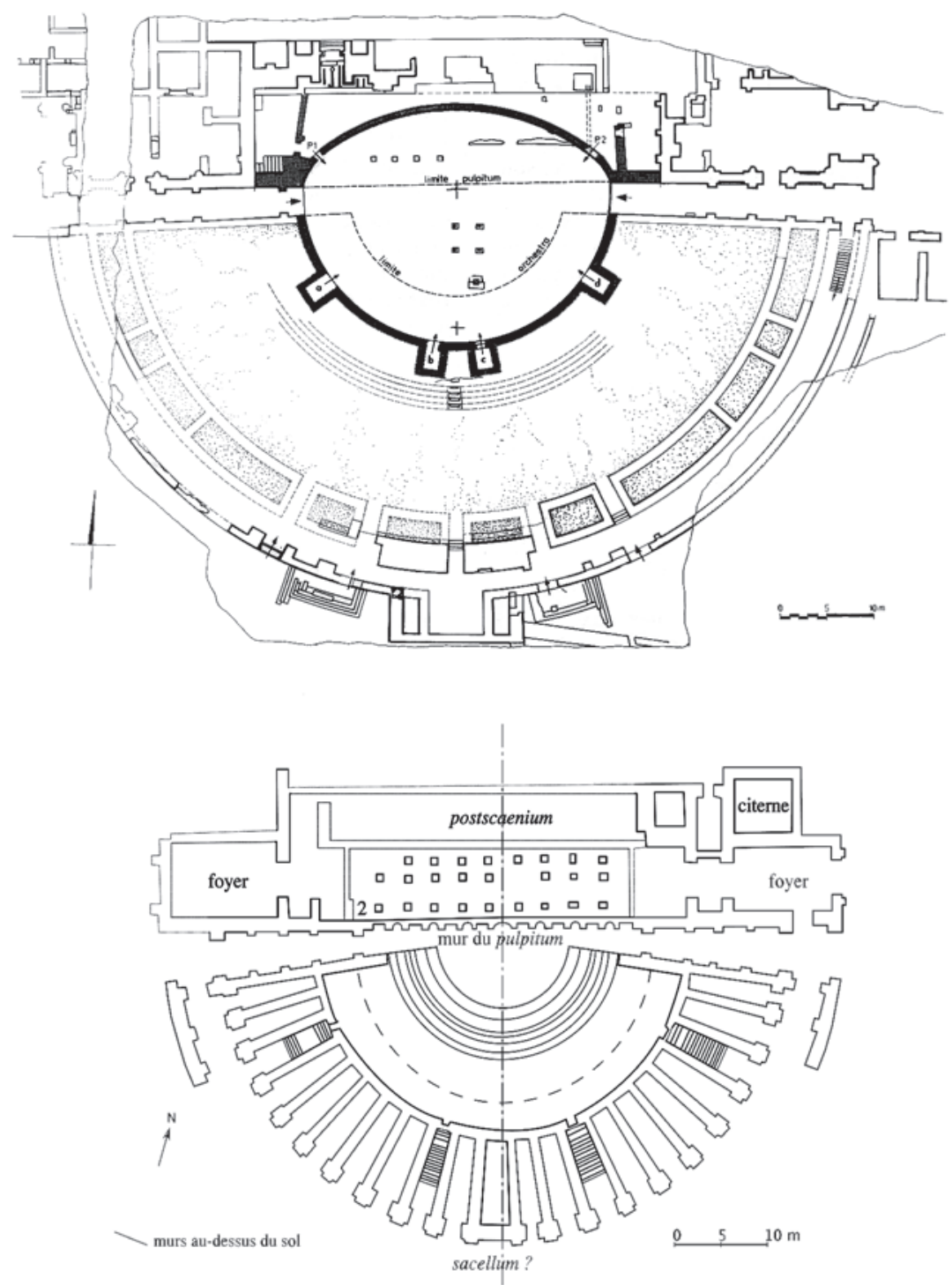

Fig. 2 - Plans des théâtres de Caesarea (d'après J.-C. Golvin) et de Tipasa à la même échelle. 


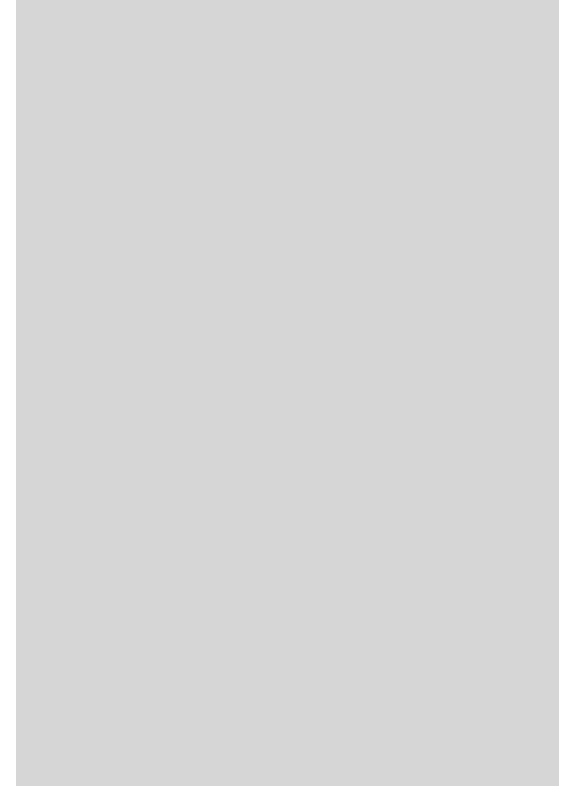

Fig. 3 - Personnification de l'Afrique. mais certainement à l'inadaptabilité de l'amphithéâtre pour les luttes de gladiateurs qui étaient de plus en plus populaires.

L'amphithéâtre, daté de la même époque que le théâtre, est en partie creusé dans le sol (fig. 4). Il a été agrandi au $\mathrm{II}^{\mathrm{e}}$ siècle de notre ère pour accueillir 15000 spectateurs. Le tracé très particulier de son arène, un espace central rectangulaire (57 $\mathrm{m}$ x $44 \mathrm{~m}$ ) prolongé par deux extrémités semi-circulaires (rayon de $22 \mathrm{~m}$ ), est tout à fait original. La longueur de l'arène le rend mieux adapté aux chasses qu'aux combats de gladiateurs ${ }^{22}$. Encore enfant, Juba II a pu assister à des jeux organisés sur les gradins temporaires en bois (spectacula) installés sur le forum romain ${ }^{23}$. Il est possible qu'il ait voulu doter sa capitale d'un amphithéâtre de caractère monumental rappelant la forme allongée de cette installation, selon la restitution de J.-C. Golvin ${ }^{24}$. Pour K. E. Welch, les spectacula ayant une forme proche de celle canonisée par les amphithéâtres monumentaux, elle propose de voir une influence du stade grec sur l'architecture de Caesarea ${ }^{25}$. En effet, l'entourage maurétanien de Juba II était fortement hellénisé. L'influence hellénistique se retrouve dans les domaines de l'architecture et de l'urbanisme en Maurétanie ${ }^{26}$.

de l'ancien amphithéâtre tombé en ruine. Ibid., p. 106.

22. J.-C. Golvin, L'amphithéatre romain, p. 113.

23. Dion Cassius, Historia romana 43.22 et $\mathrm{Ph}$. Leveau, "L'urbanisme des princes clients d'Auguste", p. 350.

24. J.-C. Golvin, L'amphithéâtre romain, p. 18-21.

25. K. E. Welch, The Roman amphitheatre, p. 47.

26. A. Pichot, Les édifices de spectacle des Maurétanies romaines, p. 192. 

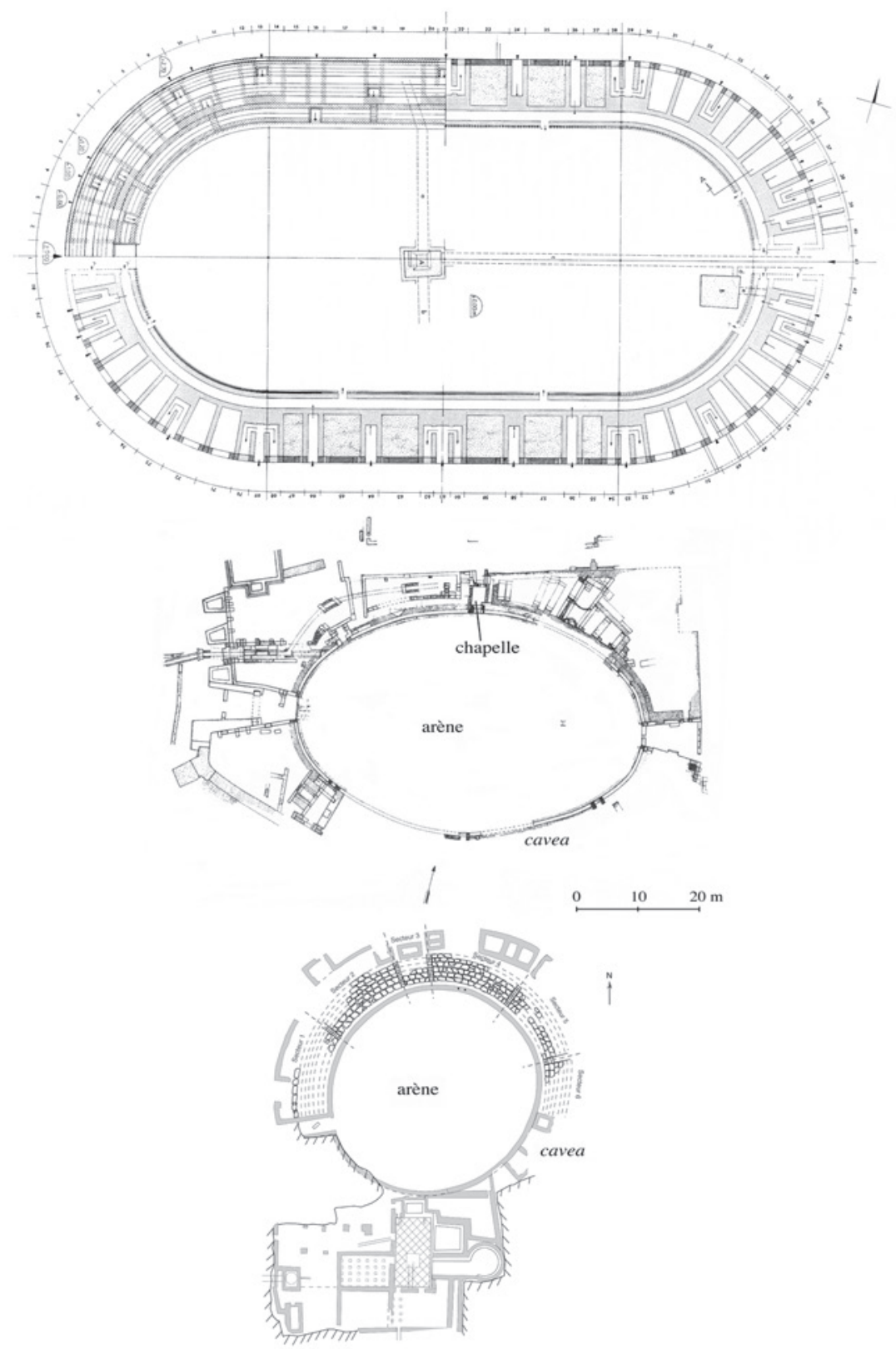

Fig. 4 - Plans des amphithéâtres de Caesarea (d'après J.-C. Golvin), de Tipasa (d'après S. Lancel) et de Lixus à la même échelle. 


\subsection{Tipasa}

Tipasa était sans doute un point de relâche. Les navigateurs phéniciens s'y ravitaillaient en eau douce et s'y arrêtaient pour la nuit ${ }^{27}$. Pline l'Ancien signale que Claude accorda à la cité un statut municipal en 46 de notre ère ${ }^{28}$. Un siècle plus tard, entre 117 et $138^{29}$, Tipasa accéda au rang de colonie romaine.

La campagne se prêtait à une exploitation fructueuse, surtout pour l'olivier, mais la ville devait sa prospérité à ses activités commerçantes, ses communications étant particulièrement bien développées grâce aux routes terrestres et maritimes ${ }^{30}$.

Le théâtre, de la fin du $\mathrm{II}^{\mathrm{e}}$ ou du début du $\mathrm{III}^{\mathrm{e}}$ siècle, fait certainement partie des travaux de prestige entrepris par la ville après avoir reçu son titre de colonie (fig. 2). Il n'est pas très bien conservé, pratiquement tous les gradins ${ }^{31}$ et le mur de scène ayant été enlevés en 1847 pour construire un hôpital.

Edmond Frézouls ${ }^{32}$ a comparé son plan aux indications de Vitruve ${ }^{33}$ : la forme semi-circulaire de l'orchestra et la largeur de la scène correspondent au De architectura, cependant la scène s'étend en profondeur aux dépens du mur de scène et ses dépendances sont particulièrement étroites. Cette hypertrophie de la scène correspond à une utilisation plus importante du plateau, puisque les spectacles romains ne se déroulaient pas dans l'orchestra. Les nombreuses niches du pulpitum et l'absence d'escalier de chaque côté se retrouvent seulement en Orient ${ }^{34}$. Ces caractéristiques architecturales sont exceptionnelles en Afrique.

L'amphithéâtre a été réalisé au III ${ }^{\mathrm{e}}$ siècle, à l'emplacement d'une ancienne nécropole en empiétant sur les bâtiments voisins ${ }^{35}$. Il a une

27. S. Gsell, Promenades archéologiques aux environs d'Alger (Cherchel, Tipasa, le Tombeau de la chrétienne), p. 85.

28. Pline l'Ancien, Histoire naturelle 5.20 et S. Lancel, Tipasa de Maurétanie, p. 17.

29. S. Lancel, "Tipasa de Maurétanie: histoire et archéologie», p. 763.

3o. Ibid., p. 764 sq.

3I. Ils font plus de $58 \mathrm{~m}$ de diamètre et pouvaient contenir 3500 personnes.

32. E. Frézouls, "Le théâtre romain de Tipasa", p. 173 sq.

33. Auteur latin du De architectura, traité d'architecture écrit au I ${ }^{\mathrm{er}}$ siècle de notre ère. Ce texte n'était pas un manuel de référence, mais la compilation de connaissances.

34. Le mur du pulpitum du théâtre de Palmyre a 13 niches et celui de Bosra 11 niches. 35. J.-C. Golvin, L'amphithéatre romain, p. 139. 
forme inhabituelle: son contour extérieur est rectangulaire et très irrégulier (fig. 4) ${ }^{36}$. L'architecte a pu être influencé par l'amphithéâtre voisin de Caesarea $^{37}$ et décider de lui donner une forme allongée.

Un mur, percé de six petites portes de service, délimitait l'arène. Les portes donnaient accès à des carceres, sauf celle située à l'extrémité du petit axe au nord qui ouvrait sur une chapelle. Un banc de pierre sur trois côtés et trois niches dans le mur opposé à la porte formaient un sacellum, où les combattants pouvaient honorer leurs dieux avant d'entrer dans l'arène ${ }^{38}$. Cette chapelle était située sous une loge et accessible depuis la rue par de petits escaliers dont les marches sont encore visibles.

\subsection{Lixus}

La fondation de Lixus par les Phéniciens (VIII ${ }^{\mathrm{e}}$-VII ${ }^{\mathrm{e}}$ siècle av. J.-C. ${ }^{39}$ ) serait antérieure à celle de Carthage. Pline l'Ancien rapporte plusieurs "récits fabuleux", même s'il ne semble guère convaincu par leur propos: "c'est là qu'on a placé le palais royal d'Antée, son combat avec Hercule et les jardins des Hespérides " ${ }^{40}$. Hercule devait pénétrer dans ce jardin dont l'entrée était gardée par un dragon pour dérober les pommes d'or. Pline voyait dans ce dragon une allégorie du fleuve Loukkos qui décrit de nombreux méandres au pied de la colline. En 42-43, Claude donna à la ville le titre de colonie romaine.

L'édifice de spectacle a été construit au cours du I ${ }^{\mathrm{er}}$ siècle apr. J.-C. ${ }^{41}$ (fig. 4). L'arène, d'une superficie de $800 \mathrm{~m}^{2}$, est pratiquement circulaire ${ }^{42}$. Elle est creusée dans la colline, jusqu'au niveau de la roche naturelle, et atteint $4 \mathrm{~m}$ de profondeur. Le mur de podium, recouvert d'un enduit peint encore visible par endroits, est conservé sur toute sa hauteur du côté des gradins, mais en grande partie ruiné de l'autre côté. Au

36. Arène: $57 \mathrm{~m} \mathrm{x} 35 \mathrm{~m}$; rectangle: $77 \mathrm{~m}$ x $55 \mathrm{~m}$.

37. D. Bomgardner, The story of the Roman amphitheatre, p. 155.

38. Ibid., p. 155 et Th. Hufschmid, Amphitheatrum in Provincia et Italia, p. 233-236 et fig. 270.

39. M. Ponsich, «Lixus: Informations archéologiques», p. 826.

40. Pline l'Ancien, Histoire naturelle 5.2-4.

4I. M. Ponsich, "Le théâtre-amphithéâtre de Lixus (Maroc)", p. 317. M. Lenoir propose une datation plus tardive, entre 70 et 120 de notre ère. M. Lenoir, "Lixus à l'époque romaine», p. 278.

42. $32,5 \mathrm{~m}$ pour le grand axe et $32 \mathrm{~m}$ pour le petit. 
sommet, en face des gradins de la quatrième région, deux blocs de calcaire sont percés de trous de poteau ${ }^{43}$. Ils pouvaient faire partie d'un système mobile de barrières en bois, mis en place lors des chasses pour éviter que les animaux n'atteignent les spectateurs; mais l'absence d'autres trous ne permet guère de soutenir cette hypothèse. Ils accueillaient peutêtre les poteaux soutenant une bannière ou un dais qui marquait une (ou des) place(s) privilégiée(s).

La grande profondeur de l'arène, qui a l'aspect d'une fosse, permettait de présenter des venationes en toute sécurité et sa forme de manège était parfaite pour des démonstrations de dressage. Les combats de gladiateurs devaient être plus rares, la fosse ne se prêtant guère à ce type de divertissement. Les combattants avaient en effet peu d'espace pour évoluer et se trouvaient rapidement coincés contre le mur.

Même si au premier abord la forme de la cavea se rapproche de celle d'un théâtre, elle occupe largement plus d'un demi-cercle et pouvait accueillir environ 300 personnes ${ }^{44}$. La pente permet de restituer des rangs au sud-est de l'arène, où les fondations de deux loges sont encore en place. Des gradins supplémentaires en bois et en matériaux périssables ont pu exister, mais il n'en reste aucune trace. Les fondations de plusieurs loges sont visibles sur le pourtour de la cavea, dans la moitié qui fait face à la porte d'entrée de l'arène, ce qui était certainement le meilleur emplacement pour suivre tout le déroulement du spectacle.

\subsection{Zilil}

La ville maurétanienne fut détruite entre 33 et 25 av. notre ère, pour installer une colonie romaine. Les Romains déplacèrent ses habitants, avec une partie de ceux de Tingi, pour fonder la colonie Iulia Ioza en Bétique $^{45}$. Quatre monuments fouillés attestent sa vitalité: le grand

43. Le bloc 1 mesure $60 \mathrm{~cm}$ x $80 \mathrm{~cm}$ avec un trou de $16 \mathrm{~cm}$ de diamètre, le bloc 2 mesure $30 \mathrm{~cm}$ x $80 \mathrm{~cm}$ avec un trou de $12 \mathrm{~cm}$ de diamètre. Les trous sont distants de $1 \mathrm{~m}$ environ.

44. Selon J.-C. Golvin (L'amphithéâtre romain, p. 233), la cavea fait $1780 \mathrm{~m}^{2}$ et pouvait contenir 4450 personnes. Les calculs par rapport au plan de M. Ponsich et aux restes en place, en accordant $40 \mathrm{~cm}$ de large par place, donnent des résultats beaucoup plus bas, plus proches de la réalité.

45. Strabon, Géographie 3.8. 
temple, les thermes, une imposante citerne et une enceinte ${ }^{46}$ de la seconde moitié du II ${ }^{\mathrm{e}}$ siècle apr. J.-C.

Cette cité romaine semble avoir été détruite entre 238 et le milieu du IV $^{\mathrm{e}}$ siècle $^{47}$. La ville fut reconstruite entre 355 et 360 , comme le montre l'étude du corpus monétaire ${ }^{48}$. Une basilique paléochrétienne, la seule dégagée au Maroc, atteste la christianisation de cette région ${ }^{49}$.

Repéré en 1842 par le consul anglais Drumond Hay, en même temps que l'ensemble des ruines romaines de Zilil, l'édifice de spectacle a été fouillé une seule fois dans les années 1880 par Henri de La Martinière:

A $130 \mathrm{~m}$ de cet édifice ${ }^{50}$ [...] se remarquent les restes très distincts du théâtre dont les dimensions sont les suivantes: $40 \mathrm{~m}$ de diamètre et $25 \mathrm{~m}$ d'ouverture; cette dernière fait face au forum. J'ai fait établir une tranchée de $2 \mathrm{~m}$ de profondeur suivant le diamètre, qui ne m’a rien révélés1.

Depuis, ce monument n'a plus jamais fait l'objet d'un programme de recherche.

Installé à l'extérieur de la cité sur une pente douce orientée nord-sud, il se situe juste en face de la porte ouest de l'enceinte. Si son élévation n'est pas bien conservée, sa forme est parfaitement visible et dessine un cercle de pratiquement $40 \mathrm{~m}$ de diamètre. L'ouverture de $25 \mathrm{~m}$ que décrit La Martinière correspond à l'espace entre les extrémités des deux tronçons de murs visibles. Ils faisaient certainement partie du mur de soutènement extérieur du bâtiment. Plusieurs gros blocs de pierre $(30 \mathrm{~cm} \mathrm{x}$ $50 \mathrm{~cm}$ ) sont visibles sur la pente nord du monument et devaient former les gradins adossés contre la colline. Aomar Akerraz propose de dater le monument de la même époque que l'enceinte, c'est-à-dire de la seconde moitié du $\mathrm{II}^{\mathrm{e}}$ siècle de notre ère ${ }^{52}$.

Il est difficile de donner plus d'informations sur ce monument tant qu'il n'est pas fouillé, surtout concernant sa fonction précise, mais nous

46. Fouillée partiellement et repérée grâce à la prospection électrique. A. Akerraz et al., "Fouilles de Dchar Jdid 1977-1980», p. 174-176.

47. Voir la dédicace à Gordien aujourd'hui disparue: CIL VIII 21818.

48. G. Depeyrot, Zilil I, p. 35-39.

49. A. Akerraz et al., «Fouilles de Dchar Jdid 1977-1980», p. 218.

50. Le grand temple.

5I. H. de La Martinière, "Notes sur les ruines de Ad Mercuri», p. 279.

52. A. Akerraz et al., «Fouilles de Dchar Jdid 1977-1980», p. 218. 
pouvons proposer quelques hypothèses de travail. Par sa forme circulaire ${ }^{53}$, il rappelle l'édifice de Lixus dont le diamètre maximum est de $50 \mathrm{~m}$. Leur orientation est identique, les gradins sont orientés vers le sud. Il pouvait servir de théâtre, mais rien ne marque la présence d'un bâtiment de scène. Sa forme accueillerait bien une petite arène ronde comme à Lixus. Le nombre de spectateurs qui pouvaient s'y installer était certainement réduit, quelques centaines tout au plus. L'édifice de Lixus et celui de Zilil semblent assez proches et on peut supposer que l'un a servi de modèle à l'autre.

\section{Quelques questions}

Après la présentation des monuments des jeux, effectuée sur la base des publications et d'une étude sur le terrain des édifices de Tingitane, nous développons deux points: la question de l'interprétation des restes de Lixus et l'importance des spectacles dans l'assimilation des Africains au mode de vie romain.

\subsection{Lixus: un édifice mixte?}

Une fois ce monument présenté comme un théâtre-amphithéâtre, rappelant ceux construits en Gaule romaine, cette définition n’a jamais été remise en doute. Maurice Euzennat a même vu dans la forme de cet édifice un théâtre de type grec, ce qu'a réfuté Michel Ponsich ${ }^{54}$. Pour ce dernier, la cavea semi-circulaire, l'arène et une scène supposée au-dessus des thermes formaient un ensemble mixte dédié au spectacle.

Pourtant, sur le terrain, aucune relation architecturale n'est visible entre les thermes et l'arène. Selon le plan publié par Michel Ponsich, le mur de podium s'appuie contre celui des thermes, mais il faudrait une fouille précise de cette partie pour le confirmer. Il est difficile de croire qu'ils étaient reliés en hauteur, aucun mur n'étant commun entre

53. Parfaitement visible sur le terrain et sur les images satellites (35०31'22'N 5०55'01'O).

54. M. Euzennat, «Héritage punique et influences gréco-romaines au Maroc, à la veille de la conquête romaine», puis la réponse de M. Ponsich, "Un théâtre grec au Maroc?». 
l'arène et les thermes au niveau des fondations. L'hypothèse d'une scène au-dessus des thermes est très fragile ${ }^{55}$. De plus, la configuration des gradins fait que seule une petite partie des spectateurs pouvait voir ce qui se déroulait sur cette scène. Les loges, censées être les meilleures places, n'y faisaient pas toutes face.

C'est bien un amphithéâtre avec sa cavea et son arène qui se trouve à Lixus, sans bâtiment de scène. Il est possible qu'un plancher ait été posé au-dessus de l'arène pour accueillir des pièces de théâtre, des mimes ou des concerts, des effets scéniques pouvant être créés avec des trappes et des monte-charges prenant appui au fond de l'arène, mais aucun édifice en dur n'accueillait ces divertissements.

Comme dernière hypothèse, prenant en compte la grande proximité entre les thermes et l'amphithéâtre, nous supposons que l'arène pouvait être utilisée comme palestre pour effectuer des exercices physiques avant de passer dans les différentes salles pour se laver ${ }^{56}$. Aucun autre espace pouvant remplir cette fonction n'est connu pour l'instant.

\subsection{Un marqueur de la romanisation des populations?}

Parmi les édifices typiques des villes romaines, les amphithéâtres et les théâtres sont les plus représentatifs de la romanité. Sur la colonne Trajane, un amphithéâtre en pierre est visible sur le panneau de la scène 30 (Pontes?) et des théâtres dans les scènes 82 (Dyrrachium) et 93 (Pontes) ${ }^{57}$. L'importance de ces édifices sur la colonne souligne leur rôle dans la symbolique des cités romaines. Elles se distinguent ainsi des villes barbares qui en sont dépourvues. Les premiers édifices de spectacle des Maurétanies et d'Afrique ont été construits à Caesarea, geste fort pour donner aux indigènes l'occasion de vivre à la romaine dans des monuments s'inspirant de ce qui se faisait de mieux à Rome. Les architectes de Tipasa les ont certainement utilisés comme exemple. Nous

55. A. Pichot, "Théâtres, amphithéâtres et cirques des Maurétanies romaines", p. 266 sq.

56. Cette hypothèse est fragile selon Y. Thébert, Thermes romains d'Afrique du Nord et leur contexte méditerranéen, p. 262.

57. G. Depeyrot, Optimo Principi, p. 16 sq. Pontes: royaume du Pont (Turquie). Dyrrachium: capitale de Nova Epirus (Albanie). Pour la scène 93, il est difficile d'identifier l'édifice: G. Depeyrot y voit un théâtre, K. E. Welch y reconnaît un amphithéâtre, K. E. Welch, The Roman amphitheatre, p. 69. 
supposons la même filiation entre les amphithéâtres de Lixus et de Zilil. Ladoption d'un mode de vie romain, pour s'attirer les bonnes grâces de l'Empire, passe par l'imitation et l'assimilation des mœurs du colonisateur. Lorsque l'on habite loin de Rome, des monuments plus proches peuvent servir de modèles. Les spectateurs qui se regroupaient pour la première fois dans ces édifices, les plus grands de la ville, avec leur architecture gigantesque et leur décor grandiose devaient être fortement impressionnés.

Au-delà de cette propagande de pierre, le public participait aux cérémonies religieuses du culte romain. Les ludi scaenici commençaient par une procession, la pompa, imitée de la pompe du cortège des triomphes organisés pour célébrer les généraux vainqueurs lors de leur retour à Rome $^{58}$. Du même type que celle qui se déroulait dans les cirques, la pompa du théâtre se terminait certainement avec le dépôt des images des dieux dans une cella, face à la scène ${ }^{59}$. Les dieux pouvaient ainsi suivre les spectacles donnés en leur honneur. Nous n’avons pas de représentation particulière ou de texte sur ces processions dans les Maurétanies, mais Ovide ${ }^{60}$ décrit une pompa circensis: les dieux les plus importants du panthéon romain étaient présentés au public et chaque spectateur, reconnaissant la divinité qu'il vénérait tout particulièrement, applaudissait davantage à son passage. Si une divinité était plus appréciée à un endroit, elle devait être mise en avant lors du défilé. Les munera commençaient hors de l'amphithéâtre avec un défilé menant en grande pompe les combattants et l'éditeur des jeux, celui qui les payait et les organisait, jusque dans l'arène. Ces processions permettaient d'intégrer totalement les divertissements dans un culte rendu aux dieux et à l'empereur.

\section{Conclusion}

Les monuments des jeux sont bien des marqueurs d'assimilation des populations à la culture romaine. Au départ, c'est l'élite étrangère qui

58. Pour plus de détails sur la pompa: Ch. Daremberg, E. Saglio, Dictionnaire des Antiquités grecques et romaines, article Circus.

59. Sur les plans des théâtres de Caesarea et de Tipasa, nous remarquons la présence d'une pièce au sommet de la cavea qui servait probablement de lieu de culte (sacellum). J. Hanson, Roman theater-temples, p. 60 et 64.

6o. Ovide, Les Amours 3.2. 
a apporté son bagage culturel, comme Juba II qui a fait revivre dans les murs de sa capitale maurétanienne les jeux de son enfance romaine. Ce mode de vie a rapidement été repris par l'élite locale puis par les populations urbaines. Les Maurétaniens furent aussi enthousiastes que le reste de l'Empire pour les ludi, les munera et les venationes. Le gouvernement romain ne pouvait qu'encourager ce mouvement, les cérémonies permettant de lier religieusement le peuple à son chef et de calmer les esprits tentés par la révolte en leur donnant du pain et des jeux ${ }^{61}$. Des théâtres furent construits plus tardivement et on fit des efforts pour continuer de les entretenir ou de les transformer afin de suivre le goût du public. Des témoignages comme celui de saint Augustin ${ }^{62}$ montrent que les théâtres étaient toujours pleins en Afrique au début du $\mathrm{IV}^{\mathrm{e}}$ siècle de notre ère.

On peut s'étonner du faible nombre d'édifices de spectacle en Tingitane. Pour certains, c'est la preuve que cette lointaine province était moins romanisée que le reste de l'Afrique. Ses relations avec la Bétique démontrent déjà que cette idée est erronée. De plus, c'est seulement au début des années 1960 que l'amphithéâtre de Lixus fut retrouvé sous l'impulsion de Michel Ponsich, qui ne pouvait comprendre qu'une ville de cette taille n'en possédât pas. Ce ne fut pas une découverte fortuite, mais dirigée qui a porté ses fruits. De récentes prospections géophysiques $^{63}$ à Volubilis n'ont pas eu les résultats attendus, mais le territoire à fouiller est vaste et les recherches sont loin d'être finies.

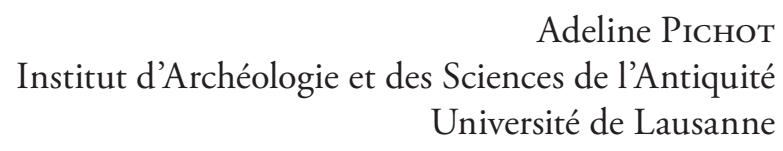

6I. Panem et circenses, Juvénal, Satires 10.81 .

62. Augustin, Les Confessions 3.1.

63. G. Ranieri et al., "Prospezione geofisica per l'individuazione di strutture archeologiche a Volubilis (Marocco)», p. 183-188. 


\section{BIBLIOGRAPHIE}

\section{Abréviations}

AE L'année épigraphique: revue des publications épigraphiques relatives à l'Antiquité romaine, Paris.

CIL Corpus inscriptionum latinarum consilio et auctoritate academiae litterarum regiae borussicae editum, Berlin, 1863-.

\section{Sources}

Augustin (Saint), Les Confessions, texte établi et traduit par P. de Labriolle, Paris, Les Belles Lettres, 2009.

Dion Cassius, Historia Romana, traduction de E. Cary, Cambridge/ London, Harvard University Press, 1954.

Juvénal, Satires, texte établi et traduit par P. de Labriolle et F. Villeneuve, Paris, Les Belles Lettres, 1967.

Ovide, Les Amours, traduit par H.Bornecque, revu par J.-P. Néraudau, introduction et notes de J.-P. Néraudau, Paris, Les Belles Lettres, 2009.

Pline l'Ancien, Histoire naturelle, Livre V, $1^{\text {re }}$ partie: 1-46 (Géographie: L'Afrique du Nord), texte établi, traduit et commenté par J. Desanges, Paris, Les Belles Lettres, 1980.

Pline l'Ancien, Histoire naturelle, Livre XVIII (De l'Agriculture), texte établi, traduit et commenté par H. Le Bonniec avec la collaboration de A. Le Bøuffle, Paris, Les Belles Lettres, 1972.

Strabon, Géographie, texte établi et traduit par F. Lasserre, Paris, Les Belles Lettres, 2003. 


\section{Etudes}

Akerraz, Aomar, El Khatib-Boujibar, Naïma, Hesnard, Antoinette, Kermorvant, Alain, Lenoir, Eliane, Lenoir, Maurice, «Fouilles de Dchar Jdid 1977-1980", Bulletin d'Archéologie Marocaine, 14 (1981-1982), p. 169-225.

Barthélemy, Suzanne, Gourevitch, Danielle, Les loisirs des Romains, Paris, Société d'édition d'enseignement supérieur, 1975.

Benseddik, Nacera, Leveau, Philippe, Ferdi, Sabah, Cherchel, Alger, Direction des Musées de l'Archéologie et des Monuments et Sites Historiques, 1983.

Benseddik, Nacera, Potter, Timothy W., La fouille du forum de Cherchel: 1977-1981, Alger, Direction de l'Archéologie, des Musées et des Monuments Historiques (Ministère de la Culture), 1993.

Blas de Robles, Jean-Marie, Sintes, Claude, Sites et monuments antiques de l'Algérie, Aix-en-Provence, Edisud, 2003.

Bomgardner, David, The story of the Roman amphitheatre, London, Routledge, 2000.

Coltelloni-Trannoy, Michèle, Le royaume de Maurétanie sous Juba II et Ptolémée (25 av. J.-C. - 40 apr. J.-C.), Paris, CNRS, 1997.

Daremberg, Charles, Saglio, Edmond, Dictionnaire des Antiquités grecques et romaines, Paris, Hachette, 1877-1919.

Decret, François, Fantar, Mhamed, L'Afrique du nord dans l'Antiquité: histoire et civilisation (des origines au Ve siècle), Paris, Payot, 1998.

Depeyrot, Georges, Zilil I. Etude du numéraire, Rome, Ecole Française de Rome, 1999.

-, Optimo Principi. Iconographie, monnaie et propagande sous Trajan, vol. II, Wetteren, Moneta, 2007.

Euzennat, Maurice, "Héritage punique et influences gréco-romaines au Maroc, à la veille de la conquête romaine", in Actes du VIII congrès international d'archéologie classique (Paris, 1963). Le rayonnement des civilisations grecque et romaine sur les cultures périphériques, Paris, De Boccard, 1965, p. 261-278.

FrÉzouls, Edmond, "Le théâtre romain de Tipasa», Mélanges de l'Ecole française de Rome - Antiquité, 91 (1952), p. 111-177.

Golvin, Jean-Claude, L'amphithéâtre romain. Essai sur la théorisation de sa forme et de ses fonctions, Paris, De Boccard, 1988. 
Golvin, Jean-Claude, Leveau, Philippe, "Lamphithéâtre et le théâtreamphithéâtre de Cherchel ", Mélanges de l'Ecole française de Rome - Antiquité, 91 (1979), p. 817-843.

Gsell, Stéphane, Promenades archéologiques aux environs d'Alger (Cherchel, Tipasa, le Tombeau de la chrétienne), Paris, Les Belles Lettres, 1926.

-, Cherchel, antique Iol-Caesarea, Alger, Direction de l'Intérieur et des Beaux-arts, 1952.

Hanson, John, Roman theater-temples, Westport, Greenwood Press, 1978.

Harmand, Louis, L'Occident romain. Gaule, Espagne, Bretagne, Afrique du Nord, Paris, Payot, 1960.

Hufschmid, Thomas, Amphitheatrum in Provincia et Italia: Architektur und Nutzung römischer Amphitheater von Augusta Raurica bis Puteoli, Augst, Pro Augusta Raurica, 2009 (Forschungen in Augst 43).

Hugoniot, Christophe, "Peut-on écrire que les spectacles furent un facteur de romanisation en Afrique du Nord?», Pallas, 68 (2005), p. 241-268.

Lachaux, Jean-Claude, Théâtres et amphithéâtres d'Afrique proconsulaire, Aix-en-Provence, Edisud, 1979.

La Martinière, Henri de, "Notes sur les ruines de Ad Mercuri", Bulletin Archéologique du Comité des Travaux Historiques et Scientifiques, 1889, p. 277-280.

Lancel, Serge, Tipasa de Maurétanie, Alger, Sous-direction des Beauxarts et Antiquités, 1971.

—, "Tipasitana V: inscriptions inédites de Tipasa», Antiquités africaines, 16 (1980), p. 135-159.

—, "Tipasa de Maurétanie: histoire et archéologie», Aufstieg und Niedergang der römischen Welt, 2.10 .2 (1982), p. 739-786.

Laporte, Jean-Pierre, «Une inscription de Saldae et la date de séparation des Maurétanies Césarienne et Sitifienne», L'Africa Romana, 12 (1998), p. 1111-1121.

Lenorr, Maurice, "Lixus à l'époque romaine", in Lixus: Actes du colloque organisé par l'Institut des sciences de l'archéologie et du patrimoine de Rabat avec le concours de l'Ecole française de Rome, Larache, 8-11 novembre 1989, Paris/Rome, De Boccard/Ecole française de Rome, 1992, p. 271-287. 
Leveau, Philippe, «L'urbanisme des princes clients d'Auguste: l'exemple de Caesarea de Maurétanie", in Architecture et société: de l'archaïsme grec à la fin de la République romaine. Actes du colloque international de Rome, 2-4 décembre 1980, Paris/Rome, CNRS/ Ecole française de Rome, 1983, p. 349-354.

PiCard, Georges Charles, "La date du théâtre de Cherchel», Bulletin d'Archéologie Algérienne, 6 (1975-1976), p. 49-54.

Ріснот, Adeline, "Théâtres, amphithéâtres et cirques des Maurétanies romaines", in L'Africa romana. Le ricchezze dell'Africa: risorse, produzioni, scambi. Atti del XVII convegno di studio Sevilla, 14-17 dicembre 2006, ed. Julián González, Roma, Carocci, 2008, p. 261-272.

—, Les édifices de spectacle des Maurétanies romaines, thèse présentée à la Faculté des lettres de l'Université de Lausanne, sous la direction du Prof. Thierry Luginbühl, 2010.

Ponsich, Michel, «Un théâtre grec au Maroc?», Bulletin d'Archéologie Marocaine, 6 (1966), p. 317-322.

—, "Le théâtre-amphithéâtre de Lixus (Maroc)», in Homenaje a García Bellido IV, Madrid, Universidad complutense de Madrid, 1979, p. 297-323.

—, "Lixus: Informations archéologiques", Aufstieg und Niedergang der römischen Welt, 10.2 (1982), p. 817-860.

Ranieri, Gaetano, El Harrouni, Khalid, Akerraz, Aomar, Deidda, Gian Piero, Deiana, Rita, Nuvoli, Marco, Erriu, Stefano, Porcu, Riccardo, "Prospezione geofisica per l'individuazione di strutture archeologiche a Volubilis (Marocco)", Rendiconti del Seminario della Facoltà di scienze dell'Università di Cagliari, 73 suppl. 1 (2003), p. 183-188.

SEAr, Frank, Roman theatres. An architectural study, Oxford, Oxford University Press, 2006.

Sintes, Claude, Rebahi, Ymouna, Algérie antique: catalogue de l'exposition, Arles, Musée de l'Arles et de la Provence antiques, 2003.

ThéBert, Yvon, Thermes romains d'Afrique du Nord et leur contexte méditerranéen, Rome, Ecole française de Rome, 2003.

WeLCH, Katherine E., The Roman amphitheatre: from its origins to the Colosseum, Cambridge, Cambridge University Press, 2007. 


\section{Crédits iconographiques}

Fig. 1:
A. Pichot.

Fig. 2:

Caesarea: Golvin, Jean-Claude, Leveau, Philippe, «L'amphithéâtre et le théâtre-amphithéâtre de Cherchel ", Mélanges de l'Ecole française de Rome - Antiquité, 91 (1979), p. 835.

Tipasa: A. Pichot d'après Frézouls, Edmond, «Le théâtre romain de Tipasa", Mélanges de l'Ecole française de Rome - Antiquité, 91 (1952), p. 114.

Fig. 3:

Blas de Robles, Jean-Marie, Sintes, Claude, Sites et monuments antiques de l'Algérie, Aix-en-Provence, Edisud, 2003, p. 42.

Fig. 4:

Caesarea: Golvin, Jean-Claude, L'amphithéâtre romain. Essai sur la théorisation de sa forme et de ses fonctions, Paris, De Boccard, 1988, pl. XXIX. Tipasa: Lancel, Serge, "Tipasitana V: inscriptions inédites de Tipasa», Antiquités africaines, 16 (1980), p. 136.

Lixus: A. Pichot d'après Ponsich, Michel, "Le théâtre-amphithéâtre de Lixus (Maroc) ", in Homenaje a García Bellido IV, Madrid, Universidad complutense de Madrid, 1979, p. 306. 